\title{
Information Degradation during Archiving in Heat-Assisted Magnetic Recording
}

\author{
T. Kobayashi, Y. Nakatani*, and Y. Fujiwara \\ Graduate School of Engineering, Mie Univ., 1577 Kurimamachiya-cho, Tsu 514-8507, Japan \\ *Graduate School of Informatics and Engineering, Univ. of Electro-Communications, 1-5-1 Chofugaoka, Chofu 182-8585, Japan
}

\begin{abstract}
We examine the dependence of information stability on certain medium parameters, namely mean Curie temperature, grain height, and anisotropy constant ratio in 4 Tbpsi heat-assisted magnetic recording, for 10 years of archiving. We introduce a minimum normalized readout field $H_{0}$ after 10 years of archiving and an information degradation rate $R_{0}$ during 10 years of archiving. To interpret the results, we investigate whether there is a correlation between $H_{0}$ or $R_{0}$ and an effective thermal stability factor for 9 and 6 grains/bit. We find that there is a strong correlation since the anisotropy constant is much larger than the shape anisotropy. The $H_{0}$ and $R_{0}$ values are functions of the effective thermal stability factor and grain number, and are independent of grain number per bit. The effective thermal stability factor necessary for no information degradation is more than about 120. Furthermore, there is a weak correlation between the initial readout field or minimum readout field after 10 years of archiving and the effective thermal stability factor.
\end{abstract}

Key words: HAMR, bit error rate, degradation rate, thermal stability factor, grain number

\section{Introduction}

The long-term stability of archived information is one of the most important properties of magnetic recording media. This stability has been widely discussed using the thermal stability factor $K_{\mathrm{u}} V /(k T)$, where $K_{\mathrm{u}}, V, k$, and $T$ are the grain anisotropy constant, grain volume, Boltzmann constant, and temperature, respectively ${ }^{1)}$. Heat-assisted magnetic recording (HAMR) is a promising candidate as a next generation magnetic recording method that can operate beyond the trilemma limit ${ }^{1)}$. HAMR is a recording method in which the medium is heated to reduce coercivity during the writing period. We have introduced an HAMR medium parameter, namely, the medium anisotropy constant ratio $K_{\mathrm{u}} / K_{\text {bulk }}{ }^{2)}$ in place of the medium anisotropy constant $K_{\mathrm{u}}$ since the $K_{\mathrm{u}}$ value at the storage temperature is a function of the medium Curie temperature $T_{\mathrm{c}}$, which is strongly related to the writing property. $K_{\mathrm{u}} / K_{\mathrm{bulk}}$ is the intrinsic ratio of the medium $K_{\mathrm{u}}$ to bulk FePt $K_{\mathrm{u}}$ regardless of $T_{\mathrm{c}}$ where FePt is a candidate HAMR medium material thanks to its large $K_{\mathrm{u}}$ and relatively low $T_{\mathrm{c}}$.

We have already calculated the numerical values of $K_{\mathrm{u}} V /(k T)$ and $K_{\mathrm{u}} / K_{\text {bulk }}$ necessary for 10 years of archiving in 2 Tbpsi HAMR by employing a bit error rate calculation using the grain error probability ${ }^{3}$. We used an error (signal) threshold of 0.5 as a calculation condition.

In this paper, we estimate the minimum normalized readout field related to the minimum error threshold, that must be readable without error after 10 years of archiving in 4 Tbpsi HAMR. Furthermore, we also

Corresponding author: T. Kobayashi (e-mail: kobayasi @phen.mie-u.ac.jp). examine the information degradation rate during 10 years of archiving. The mean Curie temperature $T_{\mathrm{cm}}$ affects various properties in HAMR. The larger grain aspect ratio $h / D_{\mathrm{m}}$ of the grain height (medium layer thickness) $h$ to the mean grain size $D_{\mathrm{m}}$ appears to make it too difficult to manufacture the medium. Furthermore, there is a concern that $K_{\mathrm{u}}$, namely, $K_{\mathrm{u}} / K_{\text {bulk }}$ decreases as $D_{\mathrm{m}}$ decreases. Thus, we examine the dependence of information stability on the medium parameters $T_{\mathrm{cm}}, h$, and $K_{\mathrm{u}} / K_{\text {bulk }}$.

\section{Calculation Method and Conditions}

\subsection{Magnetic properties}

The temperature dependence of the magnetization $M_{\mathrm{s}}$ was calculated by employing a mean field analysis4), and that of the anisotropy constant $K_{\mathrm{u}}$ was assumed to be proportional to $\left.M_{\mathrm{s}}^{25}\right) \cdot M_{\mathrm{s}}\left(T_{\mathrm{c}}, T\right)$ is a function of $T_{\mathrm{c}}$ and $T$. And $M_{\mathrm{s}}\left(T_{\mathrm{c}}=770 \mathrm{~K}, T=300 \mathrm{~K}\right)=1000 \mathrm{emu} / \mathrm{cm}^{3}$ was assumed for FePt. With this assumption, the $M_{\mathrm{S}}$ value can be calculated for all $T_{\mathrm{c}}$ and $T$ values.

The anisotropy constant $K_{\mathrm{u}}\left(T_{\mathrm{c}}, K_{\mathrm{u}} / K_{\text {bulk }}, T\right)$ is a function of $T_{\mathrm{c}}, K_{\mathrm{u}} / K_{\mathrm{bulk}}$, and $T$. And $K_{\mathrm{u}}\left(T_{\mathrm{c}}=770 \mathrm{~K}, K_{\mathrm{u}} /\right.$ $\left.K_{\text {bulk }}=1, T=300 \mathrm{~K}\right)=70 \mathrm{Merg} / \mathrm{cm}^{3}$ was assumed for bulk FePt. With this assumption, the $K_{\mathrm{u}}$ value can be calculated for all $T_{\mathrm{c}}, K_{\mathrm{u}} / K_{\text {bulk }}$, and $T$ values. No intrinsic distribution of $K_{\mathrm{u}}$ was assumed. However, there was a fluctuation in $K_{\mathrm{u}}$ caused by $T_{\mathrm{c}}$ variation.

The $T_{\mathrm{c}}$ value can be adjusted by changing the $\mathrm{Cu}$ composition $z$ for $\left(\mathrm{Fe}_{0.5} \mathrm{Pt}_{0.5}\right)_{1-z} \mathrm{Cu}_{z}$.

\subsection{Field strength}

The recording density is 4 Tbpsi, and the bit area $S$ is $161 \mathrm{~nm}^{2}$. We assumed the medium to be granular. One bit has $m$ grains in the cross-track direction and $n$ grains in the down-track direction, namely, there are $m \times n$ grains/bit. The mean grain size $D_{\mathrm{m}}$ was 
determined by

$$
D_{\mathrm{m}}=\sqrt{\frac{S}{m \times n}}-\Delta_{\mathrm{G}},
$$

where $\Delta_{\mathrm{G}}$ is the intergrain spacing. The bit aspect ratio is $D_{\mathrm{T}} / D_{\mathrm{B}}=m / n$ where $D_{\mathrm{T}}=m\left(D_{\mathrm{m}}+\Delta_{\mathrm{G}}\right)$ and $D_{\mathrm{B}}=$ $n\left(D_{\mathrm{m}}+\Delta_{\mathrm{G}}\right)$ are the track width and bit pitch, respectively. The flying height $h_{\mathrm{fly}}=4.0 \mathrm{~nm}$ is the distance between the magnetic head reader and the recording layer surface.

The magnetic field strength from the medium at the center of $m$ grains and the reader position was calculated using $m \times 30$ grains with no grain size distribution when calculating the field strength.

\subsection{Bit error rate}

The bit error rate bER was calculated by employing each grain error probability $P_{i}$,

$$
P_{i}=1-\exp \left(-f_{0} t \exp \left(-\frac{K_{\mathrm{ueff}} V}{k T}\right)\right)
$$

taking account of the shape anisotropy $M_{\mathrm{s}} H_{\mathrm{d}} / 2$ using a self-demagnetizing field $H_{\mathrm{d}}$ where $f_{0}, t=10$ years, and $K_{\text {ueff }}$ are the attempt frequency ${ }^{6)}$, time, and effective anisotropy constant, respectively. And $K_{\text {ueff }}=$ $K_{\mathrm{u}}-M_{\mathrm{s}} H_{\mathrm{d}} / 2, H_{\mathrm{d}}=8 M_{\mathrm{s}} \arctan \left(D^{2} /\left(h \sqrt{2 D^{2}+h^{2}}\right)\right), \quad V=$ $D \times D \times h$ where $D$ is the grain size. Since $H_{\mathrm{d}}$ does not affect the results as mentioned below, the magnetostatic field from surrounding grains can also be ignored. It was assumed that there is no exchange coupling between grains. For example, for 4 grains/bit, the 1 grain-error bit error rate ${ }_{4} \mathrm{bER}_{1}$ is expressed as

$$
\begin{aligned}
{ }_{4} \mathrm{bER}_{1}= & E r_{1} P_{1}\left(1-P_{2}\right)\left(1-P_{3}\right)\left(1-P_{4}\right)+\cdots \\
& +E r_{4}\left(1-P_{1}\right)\left(1-P_{2}\right)\left(1-P_{3}\right) P_{4},
\end{aligned}
$$

the 2 grain-error bit error rate ${ }_{4} \mathrm{bER}_{2}$ as

$$
\begin{aligned}
{ }_{4} \mathrm{bER}_{2}= & E_{12} P_{1} P_{2}\left(1-P_{3}\right)\left(1-P_{4}\right)+\cdots \\
& +E r_{34}\left(1-P_{1}\right)\left(1-P_{2}\right) P_{3} P_{4},
\end{aligned}
$$

the 3 grain-error bit error rate ${ }_{4} \mathrm{bER}_{3}$ as

$$
\begin{aligned}
{ }_{4} \mathrm{bER}_{3}= & E_{123} P_{1} P_{2} P_{3}\left(1-P_{4}\right)+\cdots \\
& +E r_{234}\left(1-P_{1}\right) P_{2} P_{3} P_{4},
\end{aligned}
$$

and the 4 grain-error bit error rate ${ }_{4} \mathrm{bER}_{4}$ as

$$
{ }_{4} \mathrm{bER}_{4}=P_{1} P_{2} P_{3} P_{4},
$$

where

$$
E r_{k}=1, \text { if } \frac{\sum_{i, j} M_{\mathrm{s} i j}\left(T_{\mathrm{c} i j}, 330 \mathrm{~K}\right) D_{i j}^{2}}{(m \times n) M_{\mathrm{S}}\left(T_{\mathrm{cm}}, 330 \mathrm{~K}\right) D_{\mathrm{m}}^{2}} \leq E_{\mathrm{th}},
$$

and

$$
E r_{k}=0, \text { if } \frac{\sum_{i, j} M_{\mathrm{s} i j}\left(T_{\mathrm{c} i j}, 330 \mathrm{~K}\right) D_{i j}^{2}}{(m \times n) M_{\mathrm{S}}\left(T_{\mathrm{cm}}, 330 \mathrm{~K}\right) D_{\mathrm{m}}^{2}}>E_{\mathrm{th}}
$$

Errors occur in some grains of a bit. We assumed that if the ratio of $\sum_{i, j} M_{s i j}\left(T_{c i j}, 330 \mathrm{~K}\right) D_{i j}^{2}$ to $(m \times$ $n) M_{\mathrm{s}}\left(T_{\mathrm{cm}}, 330 \mathrm{~K}\right) D_{\mathrm{m}}^{2}$ in Eqs. (7) and (8) is more than the error threshold $E_{\mathrm{th}}$, the bit is error-free after 10 years of archiving where the numerator $\sum_{i, j} M_{\mathrm{s} i j}\left(T_{\mathrm{c} i j}, 330 \mathrm{~K}\right) D_{i j}^{2}$ is the surface magnetic charge of the grains for which the magnetization turns in the recording direction, and the denominator $(m \times n) M_{\mathrm{s}}\left(T_{\mathrm{cm}}, 330 \mathrm{~K}\right) D_{\mathrm{m}}^{2}$ is the total surface magnetic charge at the readout temperature of $330 \mathrm{~K} . M_{\mathrm{s} i j}, T_{\mathrm{c} i j}$, and $D_{i j}$ are the magnetization, the Curie temperature, and the grain size of the $i j$-th grain, respectively. The total bit error rate ${ }_{4} \mathrm{bER}$ is the summation of each bit error rate ${ }_{4} \mathrm{bER}_{k}$ as follows

$$
{ }_{4} \mathrm{bER}=\Sigma_{k} \mathrm{bER}_{k} .
$$

The calculation bit number was $1 \mathrm{E}+7$. The criterion determining whether or not information is stable was assumed to be a bER of $1 \mathrm{E}-3$.

The minimum normalized readout field $H_{0}$ after 10 years of archiving can roughly be represented by $E_{\mathrm{th}}$ as

$$
H_{0}=2 E_{\mathrm{th}}-1 \text {. }
$$

We defined the information degradation rate $R_{0}$ during 10 years of archiving as

$$
R_{0}=\frac{\operatorname{bER}(10.0 \mathrm{yrs})-\mathrm{bER}(0.001 \mathrm{yrs})}{\mathrm{bER}(0.001 \mathrm{yrs})} .
$$

For example, the $R_{0}$ values are $99,9.0$, and 0.25 when the $\mathrm{bER}(0.001 \mathrm{yrs})$ values are $1 \mathrm{E}-5,1 \mathrm{E}-4$, and $8 \mathrm{E}-4$, respectively, since the $\mathrm{bER}(10.0 \mathrm{yrs})$ value is $1 \mathrm{E}-3$. The $R_{0}$ value for no information degradation is less than about 0.25 .

The conditions used when calculating the bER are summarized in Table 1. We assumed that the intergrain spacing $\Delta_{G}$ was $1.0 \mathrm{~nm}$. The grain size distribution was log-normal with a standard deviation $\sigma_{\mathrm{D}} / D_{\mathrm{m}}$ of $15 \%$, and the $T_{\mathrm{c}}$ distribution was normal with a standard deviation $\sigma_{\mathrm{Tc}} / T_{\mathrm{cm}}$ of $2 \%$. The attempt frequency $f_{0}$ is a function of the Gilbert damping constant $\alpha^{6}$, and we used an $\alpha$ value of 0.1 . The storage temperature $T_{\text {sto }}$ was $350 \mathrm{~K}$ since we take a certain margin for temperature into account.

Table 1 Conditions for bit error rate calculation.

\begin{tabular}{l|r}
\hline Recording density $(\mathrm{Tbpsi})$ & 4 \\
Intergrain spacing $\Delta_{\mathrm{G}}(\mathrm{nm})$ & 1.0 \\
Standard deviation $\sigma_{\mathrm{D}} / D_{\mathrm{m}}(\%)$ of $D_{\mathrm{m}}$ & 15 \\
Standard deviation $\sigma_{\mathrm{Tc}} / T_{\mathrm{cm}}(\%)$ of $T_{\mathrm{cm}}$ & 2 \\
Gilbert damping constant $\alpha$ & 0.1 \\
Storage temperature $T_{\text {sto }}(\mathrm{K})$ & 350 \\
\hline
\end{tabular}

\section{Calculation Results}

\subsection{Various medium parameters}

The bER value as a function $E_{\text {th }}$ and $H_{0}$ after 10 years of archiving for various $T_{\mathrm{cm}}$ values in $m \times n=$ $3 \times 3=9$ grains/bit is shown in Fig. 1 (a) where $h=10.5$ $\mathrm{nm}$ and $K_{\mathrm{u}} / K_{\text {bulk }}=0.8$. The bER value increases as the 
$T_{\mathrm{cm}}$ value decreases. There is no $E_{\mathrm{th}}$ more than 0.5 , namely, no $H_{0}$ more than 0 that satisfies bER $=1 \mathrm{E}-3$ for $T_{\mathrm{cm}}=600 \mathrm{~K}$. The time dependence of the bER is shown in Fig. 1 (b) where 0.001 years corresponds to about 9 hours. Errors increase over time for $T_{\mathrm{cm}}=650$ $\mathrm{K}$, therefore the initial bER before archiving must be low, for example, $1 \mathrm{E}-4$. On the other hand, the error scarcely increases for $T_{\mathrm{cm}}=750 \mathrm{~K}$.

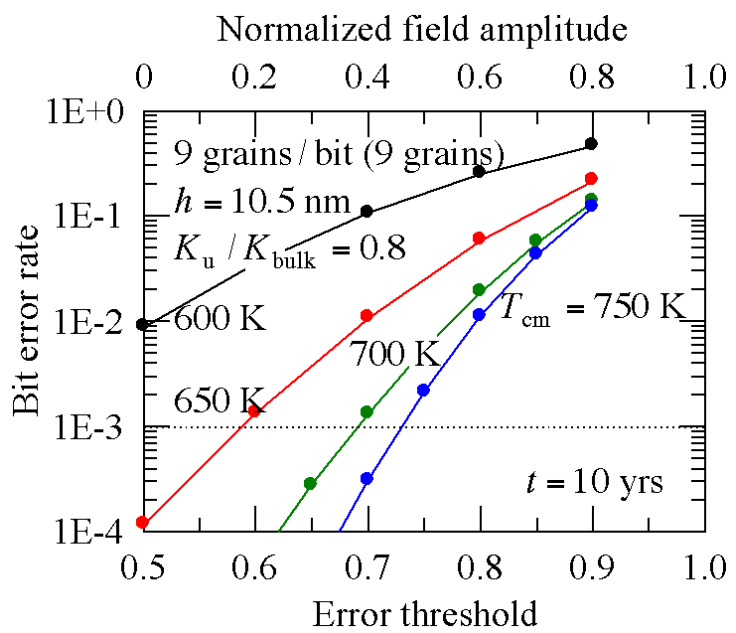

(a)

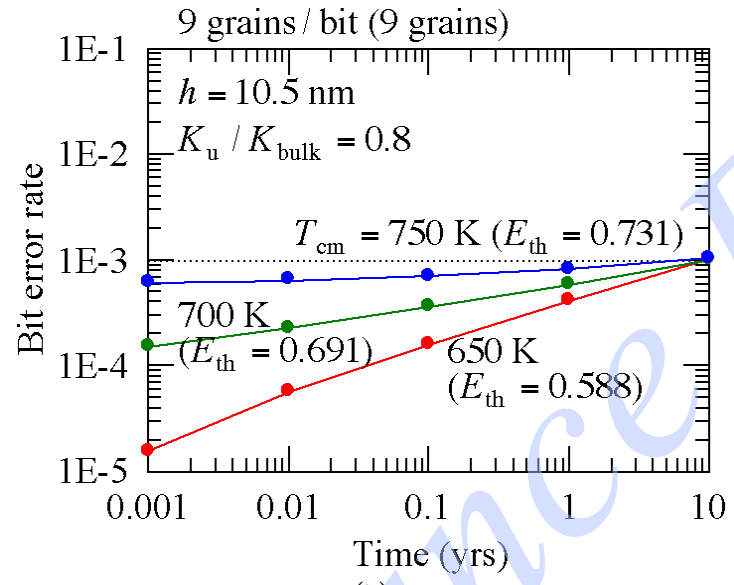

(b)

Fig. 1 (a) Bit error rate as a function of error threshold and normalized field amplitude after 10 years of archiving and (b) time dependence of bit error rate during 10 years of archiving for various mean Curie temperatures $T_{\mathrm{cm}}\left(h=10.5 \mathrm{~nm}\right.$ and $\left.K_{\mathrm{u}} / K_{\text {bulk }}=0.8\right)$.

Figure 2 shows (a) the $z$ component $H_{z}$ of the initial readout field for the down-track direction and (b) the minimum readout field $H_{0} \times H_{z}$ that must be readable without error after 10 years of archiving. Although the error scarcely increases for $T_{\mathrm{cm}}=750 \mathrm{~K}$ as shown in Fig. 1 (b), the $H_{0} \times H_{z}$ value is small as shown in Fig. 2 (b). This means that the error is caused by the cluster of small grains. The grain number per bit may increase at that time, and the $H_{0} \times H_{z}$ value may be larger. This problem is a future subject for study. In contrast, since errors increase over time for $T_{\mathrm{cm}}=650 \mathrm{~K}$ as shown in
Fig. 1 (b), the $H_{0} \times H_{z}$ value becomes small as shown in Fig. 2 (b).

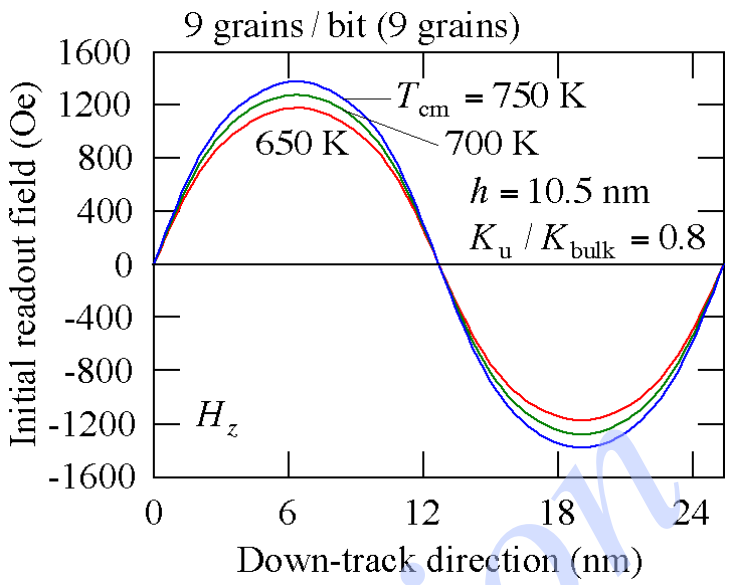

(a)

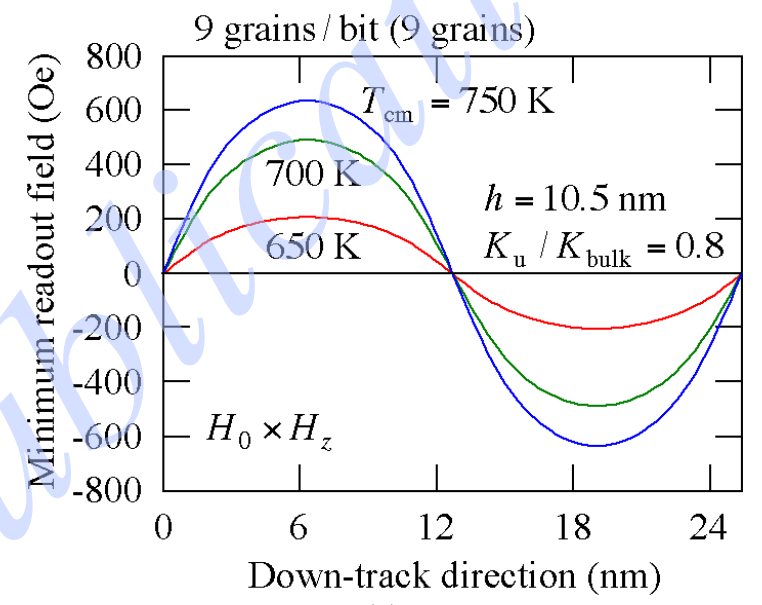

(b)

Fig. 2 (a) Initial readout field $H_{z}$ and (b) minimum readout field $H_{0} \times H_{z}$ after 10 years of archiving for various mean Curie temperatures $T_{\mathrm{cm}}(h=10.5 \mathrm{~nm}$ and $\left.K_{\mathrm{u}} / K_{\text {bulk }}=0.8\right)$.

If $T_{\mathrm{cm}}=750 \mathrm{~K}, h=6.5 \mathrm{~nm}$, and $K_{\mathrm{u}} / K_{\mathrm{bulk}}=0.8$, or if $T_{\mathrm{cm}}=750 \mathrm{~K}, h=10.5 \mathrm{~nm}$, and $K_{\mathrm{u}} / K_{\mathrm{bulk}}=0.5$, there is no $E_{\text {th }}$ more than 0.5 , or no $H_{0}$ more than 0 that satisfies bER $=1 \mathrm{E}-3$.

The results for various medium parameters, namely $T_{\mathrm{cm}}, h$, and $K_{\mathrm{u}} / K_{\text {bulk }}$, are summarized in Tables 2 and 3 for $m \times n=3 \times 3=9$ grains/bit and $m \times n=3 \times 2=6$ grains/bit, respectively. $H_{z \text { peak }}$ is the peak $Z$ component $H_{z}$ of the initial readout field, and $H_{0} \times$ $H_{z \text { zeak }}$ is the minimum peak readout field that must be readable without error after 10 years of archiving. When adjacent track interference is considered, some grains are used as a guard band. Therefore, we also examined $H_{0}, R_{0}, H_{\text {zpeak }}$, and $H_{0} \times H_{\text {zpeak }}$ for $m \times n=2 \times 3=6$ grains in 9 grains/bit and $m \times n=2 \times 2=4$ grains in 6 grains/bit.

The information during 10 years of archiving is stable for 9 grains/bit media with $T_{\mathrm{cm}}=750 \mathrm{~K}, h=10.5 \mathrm{~nm}$, and $K_{\mathrm{u}} / K_{\text {bulk }}=0.8$ as shown in Table 2 . 
Table 2 Minimum normalized readout field $H_{0}$, information degradation rate $R_{0}$, initial readout field $H_{\text {zpeak }}$, and minimum readout field $H_{0} \times H_{\text {zpeak }}$ after 10 years of archiving for 9 and 6 grains in 9 grains/bit.

\begin{tabular}{l|r|r|r|r|r|r|r}
\hline 9 grains / bit & \multicolumn{6}{|c}{$D_{\mathrm{m}}=3.2 \mathrm{~nm}$} \\
\hline$T_{\text {cm }}(\mathrm{K})$ & 750 & 700 & 650 & 750 & 750 & 750 & 750 \\
$h(\mathrm{~nm})$ & 10.5 & 10.5 & 10.5 & 8.5 & 7.5 & 10.5 & 10.5 \\
$K_{\mathrm{u}} / K_{\text {bulk }}$ & 0.8 & 0.8 & 0.8 & 0.8 & 0.8 & 0.7 & 0.6 \\
$h / D_{\mathrm{m}}$ & 3.2 & 3.2 & 3.2 & 2.6 & 2.3 & 3.2 & 3.2 \\
$K_{\text {ueff }} V /(k T)$ & 113 & 96 & 79 & 91 & 80 & 99 & 84 \\
$(T=350 \mathrm{~K})$ & & & & & & & \\
9 grains & & & & & & & \\
$H_{0}$ & 0.46 & 0.38 & 0.18 & 0.34 & 0.19 & 0.40 & 0.27 \\
$R_{0}$ & 0.71 & 5.8 & 64 & 12 & 47 & 4.0 & 26 \\
$H_{\text {zpeak }}(\mathrm{Oe})$ & 1378 & 1278 & 1173 & 1320 & 1276 & 1378 & 1378 \\
$H_{0} \times H_{\text {zpeak }}$ & 636 & 489 & 206 & 450 & 240 & 558 & 366 \\
$\quad(\mathrm{Oe})$ & & & & & & & \\
6 grains & & & & & & & \\
$H_{0}$ & 0.34 & 0.23 & $\mathrm{n} / \mathrm{a}$ & 0.18 & $\mathrm{n} / \mathrm{a}$ & 0.26 & 0.08 \\
$R_{0}$ & 1.2 & 8.4 & $\mathrm{n} / \mathrm{a}$ & 15 & $\mathrm{n} / \mathrm{a}$ & 6.0 & 28 \\
$H_{\text {zpeak }}($ Oe) $)$ & 1190 & 1103 & 1012 & 1146 & 1112 & 1190 & 1190 \\
$H_{0} \times H_{\text {zpeak }}$ & 405 & 254 & $\mathrm{n} / \mathrm{a}$ & 201 & $\mathrm{n} / \mathrm{a}$ & 308 & 96 \\
$($ Oe $)$ & & & & & & & \\
\hline
\end{tabular}

Table 3 Minimum normalized readout field $H_{0}$, information degradation rate $R_{0}$, initial readout field $H_{\text {zpeak }}$, and minimum readout field $H_{0} \times H_{\text {zpeak }}$ after 10 years of archiving for 6 and 4 grains in 6 grains/bit.

\begin{tabular}{l|r|r|r|r|r|r}
\hline 6 grains / bit & \multicolumn{5}{|c}{$D_{\mathrm{m}}=4.2 \mathrm{~nm}$} \\
\hline$T_{\mathrm{cm}}(\mathrm{K})$ & 600 & 750 & 750 & 650 & 650 & 750 \\
$h(\mathrm{~nm})$ & 10.5 & 6.5 & 10.5 & 7.5 & 10.5 & 7.5 \\
$K_{\mathrm{u}} / K_{\text {bulk }}$ & 0.8 & 0.8 & 0.5 & 0.8 & 0.6 & 0.6 \\
$h / D_{\mathrm{m}}$ & 2.5 & 1.6 & 2.5 & 1.8 & 2.5 & 1.8 \\
$K_{\text {ueff }} V /(k T)$ & 107 & 115 & 117 & 94 & 99 & 100 \\
$(T=350 \mathrm{~K})$ & & & & & & \\
6 grains & & & & & & \\
$H_{0}$ & 0.31 & 0.35 & 0.36 & 0.21 & 0.27 & 0.27 \\
$\quad R_{0}$ & 2.3 & 0.82 & 0.63 & 9.8 & 5.6 & 5.0 \\
$H_{\text {zpeak }}(\mathrm{Oe})$ & 1057 & 1240 & 1371 & 1098 & 1167 & 1290 \\
$H_{0} \times H_{\text {zpeak }}($ Oe $)$ & 331 & 434 & 491 & 236 & 313 & 353 \\
4 grains & & & & & & \\
$H_{0}$ & 0.14 & 0.21 & 0.22 & 0.02 & 0.08 & 0.09 \\
$R_{0}$ & 4.9 & 1.6 & 1.2 & 9.1 & 8.0 & 7.8 \\
$H_{\text {zpeak }}(\mathrm{Oe})$ & 950 & 1127 & 1232 & 994 & 1049 & 1168 \\
$H_{0} \times H_{\text {zpeak }}($ Oe $)$ & 136 & 231 & 268 & 23 & 86 & 103 \\
\hline
\end{tabular}

Although the $T_{\mathrm{cm}}, h$, and $K_{\mathrm{u}} / K_{\text {bulk }}$ values can be reduced to $700 \mathrm{~K}, 8.5 \mathrm{~nm}$, and 0.6 , respectively, simultaneous reduction cannot be realized. The $h / D_{\mathrm{m}}$ value necessary for information stability is especially large due to the small grain size $D_{\mathrm{m}}$ of $3.2 \mathrm{~nm}$. The medium parameters to be reduced must be selected depending on the difficulty of manufacturing the medium.

Since the mean grain size $D_{\mathrm{m}}$ for 6 grains/bit increases to $4.2 \mathrm{~nm}$ from $3.2 \mathrm{~nm}$ in 9 grains/bit as shown in Table 3, the degree of freedom in design increases. The grain area $D_{\mathrm{m}}^{2}$ ratio is 1.68 and more than $9 / 6=1.5$ between 6 and 9 grains/bit since the $\Delta_{G}$ value is independent of the grain number per bit.

\subsection{Effective thermal stability factor}

To enable us to interpret the above calculated results, we plot the $H_{0}$ and $R_{0}$ values against

$$
K_{\text {ueff }} V /(k T)=\left(K_{\mathrm{u}}-M_{\mathrm{s}} H_{\mathrm{d}} / 2\right)\left(D_{\mathrm{m}} \times D_{\mathrm{m}} \times h\right) /(k T)
$$

at $350 \mathrm{~K}$ in Figs. 3 and 4 for 9 and 6 grains/bit, respectively. All the calculated data for 9 and 6 grains in 9 grains/bit and for 6 and 4 grains in 6 grains/bit are on their lines. Furthermore, the $H_{0}$ and $R_{0}$ values for 6 grains in 9 and 6 grains/bit are the same as shown in Figs. 4 (a) and (b), respectively. $H_{0}$ and $R_{0}$ are functions of $K_{\text {ueff }} V /(k T)$ and grain number, and are independent of grain number per bit since $K_{\mathrm{u}} \gg M_{\mathrm{s}} H_{\mathrm{d}} / 2$ in $K_{\text {ueff }}$.

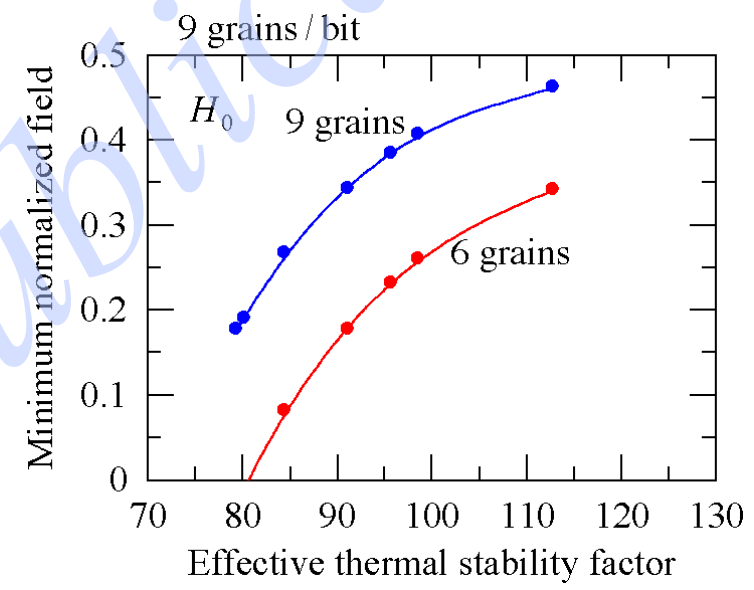

(a)

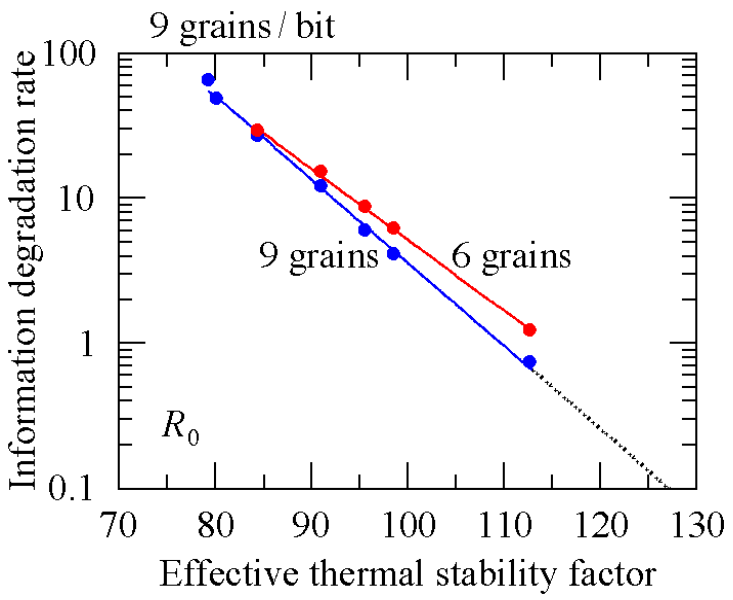

(b)

Fig. 3 (a) Minimum normalized readout field $H_{0}$ and (b) information degradation rate $R_{0}$ against effective thermal stability factor $K_{\text {ueff }} V /(k T)$ for 9 and 6 grains in 9 grains/bit. 


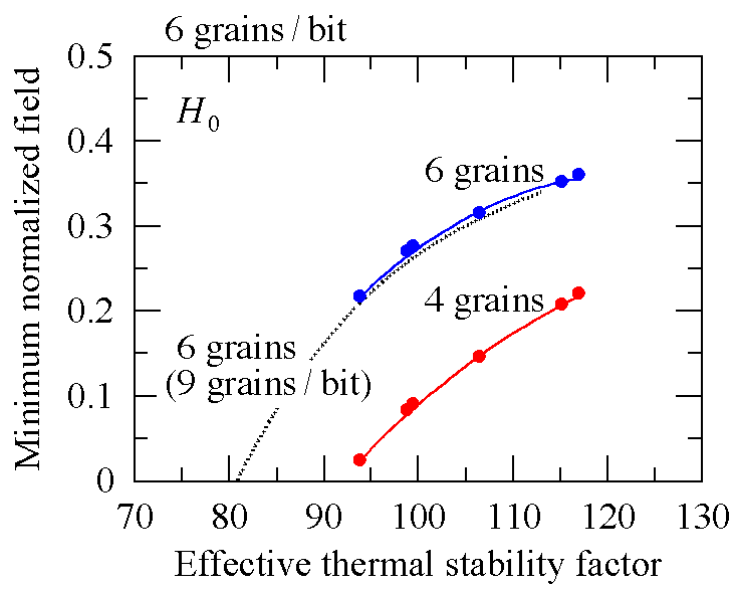

(a)

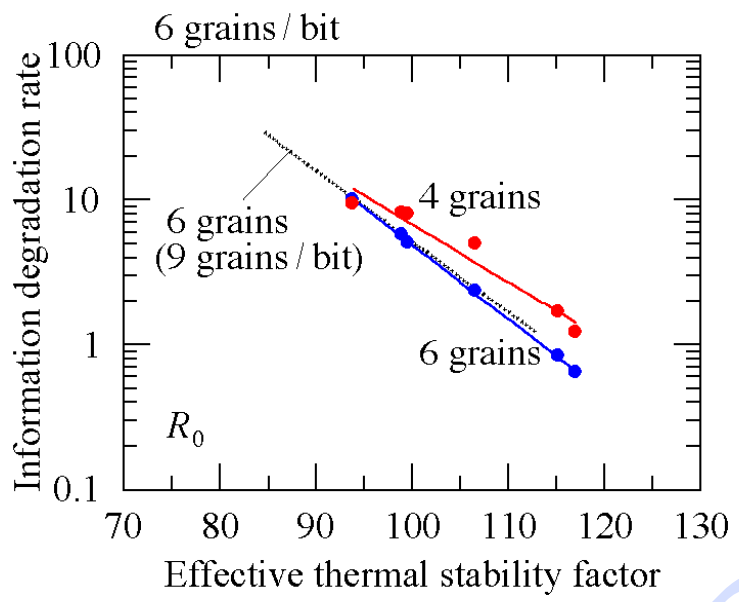

(b)

Fig. 4 (a) Minimum normalized readout field $H_{0}$ and (b) information degradation rate $R_{0}$ against effective thermal stability factor $K_{\text {ueff }} V /(k T)$ for 6 and 4 grains in 6 grains/bit.

The $H_{0}$ value for 9 grains is larger than that for 6 grains in 9 grains/bit as shown in Fig. 3 (a) due to a statistical factor. The statistical factor can be explained using the following example. If one bit contains many grains, the bER becomes low since the probability of a simultaneous error is very low for more than half of the grains in one bit. For example, when all the grains are assumed to be homogeneous, and $P$ and $E_{\text {th }}$ are 0.1 and 0.5 , respectively, the bER calculated for 5 grains/bit is

$$
\mathrm{bER}=\sum_{k=3}^{5}\left(\begin{array}{l}
5 \\
k
\end{array}\right) P^{k}(1-P)^{5-k} \approx 0.009 .
$$

On the other hand, the bER for 9 grains/bit is

$$
\mathrm{bER}=\sum_{k=5}^{9}\left(\begin{array}{l}
9 \\
k
\end{array}\right) P^{k}(1-P)^{9-k} \approx 0.0009,
$$

which is much lower than that for 5 grains/bit. Therefore, to obtain the same bER, $P$ must be reduced for 5 grains/bit, and a larger $K_{\text {ueff }} V /(k T)$ value is necessary.

And the $H_{0}$ value for 6 grains in 9 grains/bit is larger than that for 4 grains in 6 grains/bit as shown in Fig. 4 (a). Although it is necessary to increase the $K_{\text {ueff }} V /(k T)$ value for 6 grains/bit to obtain the same $H_{0}$ value for 9 grains/bit, it is easy to increase the $K_{\text {ueff }} V /(k T)$ value for 6 grains/bit since the one grain area is 1.68 times larger. However, there is a concern that the readout SNR for 6 grains/bit is smaller than that for 9 grains/bit due to the small grain number.

A linear relationship was found between logarithmic $R_{0}$ and $K_{\text {ueff }} V /(k T)$, and the $R_{0}$ value decreases slightly in the order of 4, 6, and 9 grains as shown in Figs. 3 (b) and $4(\mathrm{~b})$. The $K_{\text {ueff }} V /(k T)$ value necessary for no information degradation is more than about 120 .

The $H_{\text {zpeak }}$ and $H_{0} \times H_{\text {zpeak }}$ values are plotted against $K_{\text {ueff }} V /(k T)$ in Figs. 5 and 6 for 9 and 6 grains/bit, respectively. The lines in the figures are guides for the eye. The cause of data scattering is that $H_{0}$ is a function of $K_{\text {ueff }} V /(k T)$, and $H_{\text {zpeak }}$ is a function of $M_{\mathrm{s}}, D_{\mathrm{m}}$, and $h$. And although the $M_{\mathrm{s}}$ value is a function of $T_{\mathrm{c}}$, it is independent of $K_{\mathrm{u}} / K_{\text {bulk }}$. Roughly, all calculation data for 9 and 6 grains in 9 grains/bit and for 6 and 4 grains in 6 grains/bit are on their lines as shown in Figs. 5 and 6, respectively.

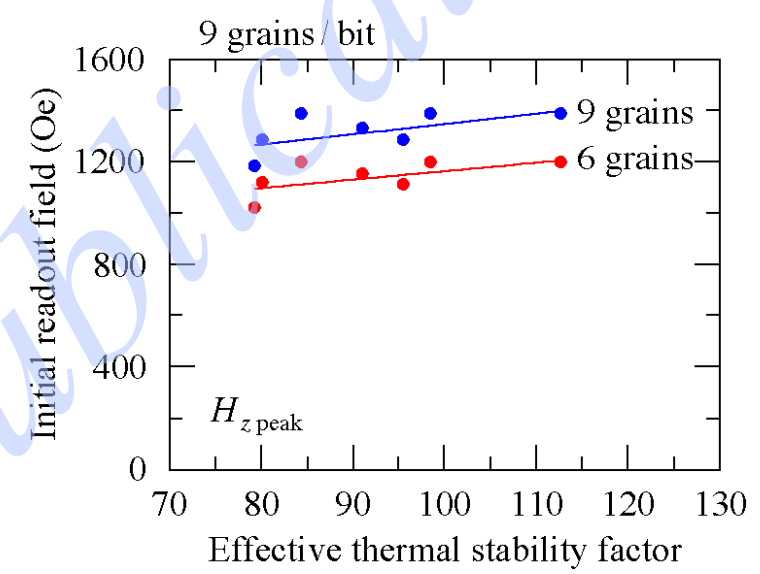

(a)

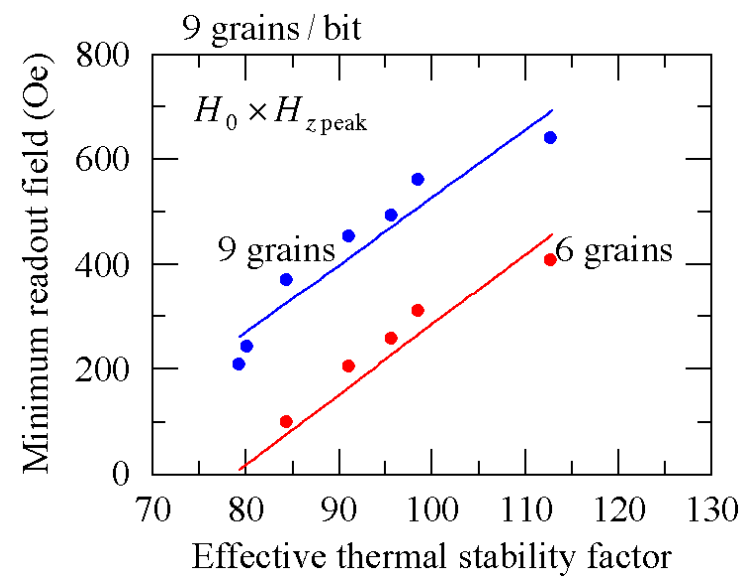

(b)

Fig. 5 (a) Initial readout field $H_{z \text { peak }}$ and (b) minimum readout field $H_{0} \times H_{z \text { peak }}$ against effective thermal stability factor $K_{\text {ueff }} V /(k T)$ for 9 and 6 grains in 9 grains/bit. 


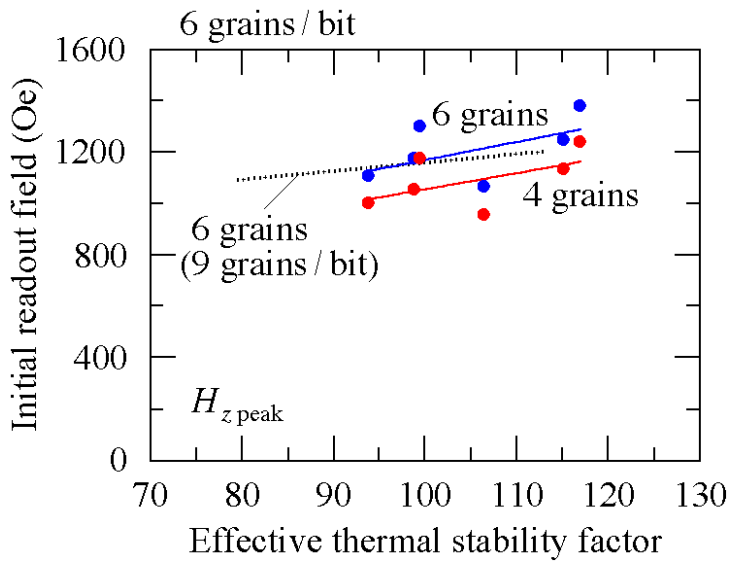

(a)

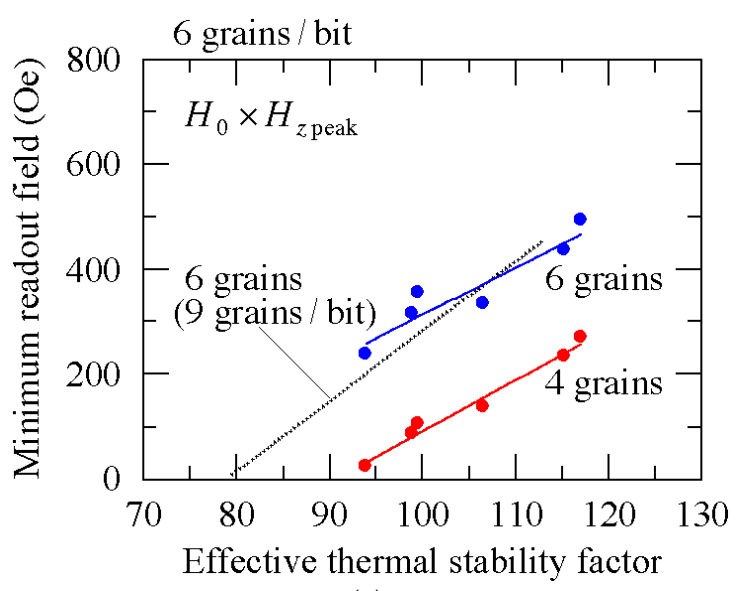

(b)

Fig. 6 (a) Initial readout field $H_{z \text { peak }}$ and (b) minimum readout field $H_{0} \times H_{z \text { peak }}$ against effective thermal stability factor $K_{\text {ueff }} V /(k T)$ for 6 and 4 grains in 6 grains/bit.

The $H_{0} \times H_{\text {zpeak }}$ value for 9 grains is larger than that for 6 grains in 9 grains/bit as shown in Fig. 5 (b) since both $H_{0}$ and $H_{z \text { peak }}$ for 9 grains are larger than those for 6 grains as shown in Figs. 3 (a) and 5 (a). Furthermore, the $H_{0} \times H_{\text {zpeak }}$ values for 6 grains in 9 and 6 grains/bit are roughly the same as shown in Fig. 6 (b).

There are many factors to be considered when designing the medium. A high $T_{\mathrm{cm}}$ is disadvantageous in terms of the heat resistance of the writing head and/or the surface lubricant. On the other hand, a low $T_{\mathrm{cm}}$ is disadvantageous in terms of information stability for 10 years of archiving since $K_{\mathrm{u}}$ at the storage temperature becomes smalli). A low $T_{\mathrm{cm}}$ is also disadvantageous since the thermal gradient becomes small ${ }^{7}$. A larger $h / D_{\mathrm{m}}$ seems to make it too difficult to manufacture the medium. There is a concern that $K_{\mathrm{u}}$ decreases as $D_{\mathrm{m}}$ decreases.
We must comprehensively examine all the factors if we are to determine the medium parameters. Since these factors are currently being experimentally investigated by many researchers, it is currently impossible to determine the medium parameters. However, the above calculations will be useful for determining them in the future.

\section{Conclusions}

We examined the dependence of information stability for 10 years of archiving on various medium parameters, namely $T_{\mathrm{cm}}, h$, and $K_{\mathrm{u}} / K_{\text {bulk }}$, in 4 Tbpsi HAMR. As a result, we found that the minimum normalized readout field $H_{0}$ and information degradation rate $R_{0}$ are functions of the effective thermal stability factor $K_{\text {ueff }} V /(k T)$ and grain number, and are independent of grain number per bit since $K_{\mathrm{u}} \gg M_{\mathrm{s}} H_{\mathrm{d}} / 2$ in $K_{\text {ueff. The }}$ $K_{\text {ueff }} V /(k T)$ value necessary for no information degradation is more than about 120 . Furthermore, there is a weak correlation between the initial readout field $H_{\text {zpeak }}$ or the minimum readout field $H_{0} \times H_{\text {zpeak }}$ and $K_{\text {ueff }} V /(k T)$.

Although the $T_{\mathrm{cm}}, h$, and $K_{\mathrm{u}} / K_{\text {bulk }}$ values can be reduced to $700 \mathrm{~K}, 8.5 \mathrm{~nm}$, and 0.6 , respectively, for 9 grains/bit, a simultaneous reduction cannot be realized. The $h / D_{\mathrm{m}}$ value necessary for information stability is especially large due to the small grain size $D_{\mathrm{m}}$ of $3.2 \mathrm{~nm}$. Depending on the difficulty involved in manufacturing a medium, we must select the medium parameters to be reduced.

It is easy to increase the $K_{\text {ueff }} V /(k T)$ value for 6 grains/bit since the one grain area is 1.68 times larger.

Acknowledgement We acknowledge the support of the Advanced Storage Research Consortium (ASRC), Japan.

\section{References}

1) S. H. Charap, P. -L. Lu, and Y. He: IEEE Trans. Magn., 33, 978 (1997).

2) T. Kobayashi, Y. Isowaki, and Y. Fujiwara: J. Magn. Soc. Jpn., 39, 8 (2015)

3) T. Kobayashi, Y. Nakatani, and Y. Fujiwara: J. Magn. Soc. Jpn., 43, 114 (2019).

4) M. Mansuripur and M. F. Ruane: IEEE Trans. Magn., MAG22, 33 (1986).

5) J. -U. Thiele, K. R. Coffey, M. F. Toney, J. A. Hedstrom, and A. J. Kellock: J. Appl. Phys., 91, 6595 (2002).

6) E. D. Boerner and H. N. Bertram: IEEE Trans. Magn., 34, 1678 (1998).

7) T. Kobayashi, Y. Nakatani, and Y. Fujiwara: J. Magn. Soc. Jpn., 44, 122 (2020).

Received Oct. 8, 2021; Accepted Oct. 26, 2021 